Baseline bloods (or declined) $=89 \%$, Baseline ECG (or declined $)=67 \%$

Complete monitoring bloods $=44 \%$, Physical health monitoring parameters complete $=56 \%$

Monitoring schedule present in notes and current $=38 \%$, Present, not current $=50 \%$ ( $0 \%$ on PARIS).

Conclusion. Lower numbers at re-audit limit interpretation.

Further recommendations: Antipsychotic initiation checklist; Central bloods diary for clinicians; Antipsychotic care-pathway booklet, co-produced with young people, incorporating the monitoring schedule.

\section{Characterising a cohort of patients referred to a liaison psychiatry service from the Intensive Care Unit}

Jennifer Hanks*, Chun Chiang Sin Fai Lam, Isabel McMullen and Martin Parsons

South London and Maudsley NHS Foundation Trust

${ }^{*}$ Corresponding author.

doi: 10.1192/bjo.2021.852

Aims. This is descriptive study of a cohort of patients referred to a liaison psychiatry service from the intensive care department of a major London teaching hospital and trauma centre. The objective was to characterise key patterns in reasons for referral, nature of input, and gain a general sense of the workload. The rationale for collating this information was the consideration to developing a specific intensive care liaison service given the increasing evidence about the cognitive and mental health impacts of postintensive care syndrome and the need for a coordinated management approach between stakeholders.

Method. A cohort of 80 patients referred to liaison psychiatry service over a 6-month period from May to October 2020 was used. Descriptive statistics were used to characterise the patient's age, referring ward, reason for admission and referral, nature of input, number of reviews, previous engagement with mental health services, whether substance abuse or self-harm were related to the admission, and the destination upon discharge.

Result. The age range of patients at point of referral was 25-80 years. For $25 \%$ of patients, this admission marked their first engagement with secondary mental health services and for around $50 \%$, not only was a new diagnosis given during the admission, but there was no recorded history of any psychiatric diagnoses. Around $10 \%$ of patients were referred for management of delirium. Anxiety disorder accounted for the greatest proportion of diagnoses upon discharge, at $22 \%$. There was much variability in the number of intensive care ward reviews carried out, ranging from one to over 10 .

In $24 \%$, self-harm led to presentation and $18 \%$ had comorbid substance misuse. Medication review was the single most common reason for referral in $13 \%$, whereas requests for talking therapy and capacity assessments were $5 \%$ and $2 \%$ respectively. The vast majority of patients required a level of ongoing psychiatric input warranting community involvement or admission.

Conclusion. This cohort often required detailed work-ups, new diagnoses and a high level of subsequent psychiatric management following discharge from hospital. The wide age range of patients meant that both working age and older adult liaison teams were involved in assessing referrals. Consideration could be given to a specific intensive-care liaison service due to the workload and complexity of needs, as well as the increasing awareness of the need for family support and early inclusion both for their benefit and that of the patient, particularly when the proportion of new diagnoses in this cohort is considered.
Clinical audit on possible causes of hospital initiated clinic cancellations and recommendation to improve the service

Tanzida Haque ${ }^{1 \star}$, Mosab Mohammed Jodat Ibrahim ${ }^{2}$ and Bapu Ravindranath ${ }^{3}$

${ }^{1}$ Mersey Care NHS Foundation Trust, Merseycare NHS Foundation Trust; ${ }^{2}$ Mersey Care NHS Foundation Trust, Merseycare NHS Foundation Trust, HEE NW and ${ }^{3}$ Mersey Care NHS Foundation Trust

${ }^{\star}$ Corresponding author.

doi: 10.1192/bjo.2021.853

Aims. The aim of this audit is to explore the possible causes of clinic cancellation in an inner city CMHT and the recommendation to reduce the burden.

Background. Cancellations of planned appointments have been a major and long-standing problem for healthcare organisations across the world. It represents a significant loss of revenue and waste of resources, have significant psychological, social and financial implications for patients and their families and represent a significant loss of training opportunities for trainees. Re-scheduling appointment is one of the major issues of inconvenience to the patients. It also increases workload for the patient appointment team.

Method. Data have been collected retrospectively from patient appointment booking team regarding clinic cancellation with causes of cancellation recorded in the system (01/07/2019-30/ 09/2019). The investigators have investigated if the cancellation has been made when it was absolutely necessary to cancel the clinic (Unavailability of doctors due to leave/on calls) and if patients have been informed at least 8 weeks prior to the appointed clinic as per trust protocol.

Result. Total number of 193 clinics were booked at the CMHT from July 2019 - September 2019. About 54\% clinics were cancelled during the time period. The Clinic Cancellation rate was higher in September (68\%) and was lowest in August (30.30\%). As the month of July is the changeover period for trainees, the number of clinics booked during August was relatively less than normal. $72 \%$ clinics were cancelled by junior doctors and $28 \%$ clinics were cancelled by consultants at the CMHT. The major cause of clinic cancellation was unavailability of the junior doctors due to on call $(31.58 \%)$ which was not communicated to the patient appointment booking team. Due to annual leave, $25 \%$ clinics were cancelled and $21 \%$ clinics were cancelled due to study leave. In both cases it is evident that, lack of communication between clinicians and patient appointment team are primarily responsible for hospital-initiated clinic cancellations. As per Patient Appointment booking team, around 50\% cases, patients were informed 8 weeks in advance before cancelling the clinics.

Conclusion. This is evident from this audit that the number of hospital-initiated clinic cancellations can be reduced by improving communication between Patient Appointment booking service, Medical staffing department and clinicians. The findings of the audit have been shared locally with CMHT managers, clinicians and with the patient appointment booking team.

\section{Management of emotionally unstable personality disorder in an urban Irish setting}

Calvin Har Siu Yee*

St. Vincent's Hospital

${ }^{*}$ Corresponding author.

doi: 10.1192/bjo.2021.854 